\title{
Blow-up and non-extinction for a nonlocal parabolic equation with logarithmic nonlinearity
}

\author{
Lijun Yan ${ }^{1}$ and Zuodong Yang ${ }^{2^{*}}$ (I)
}

"Correspondence:
zdyang_jin@263.net
${ }^{2}$ School of Teacher Education,
Nanjing Normal University, Nanjing,
China
Full list of author information is
available at the end of the article

available at the end of the article

\begin{abstract}
This paper is devoted to studying a nonlocal parabolic equation with logarithmic nonlinearity $u \log |u|-f_{\Omega} u \log |u| d x$ in a bounded domain, subject to homogeneous Neumann boundary value condition. By using the logarithmic Sobolev inequality and energy estimate methods, we get the results under appropriate conditions on blow-up and non-extinction of the solutions, which extend some recent results.
\end{abstract}

Keywords: Blow-up; Non-extinction; Nonlocal parabolic equation

\section{Introduction}

In this paper, we consider the Neumann problem to the following parabolic equation:

$$
\begin{cases}u_{t}-\Delta u=u \log |u|-f_{\Omega} u \log |u| d x, & x \in \Omega, t>0, \\ \frac{\partial u}{\partial n}=0, & x \in \partial \Omega, t>0, \\ u(x, 0)=u_{0}(x), & x \in \Omega,\end{cases}
$$

where $\Omega \subset \mathbf{R}^{n}$ is a bounded domain with smooth boundary, $f_{\Omega} u_{0} d x=\frac{1}{|\Omega|} \int_{\Omega} u_{0} d x=0$ with $u_{0} \not \equiv 0$. It is easy to check that the solution $u$ of (1.1) is $L^{1}$-conservative and hence

$$
\int_{\Omega} u d x=\int_{\Omega} u_{0} d x=0
$$

In fact, problem (1.1) has been studied by many other authors in a more general form:

$$
\begin{cases}u_{t}-\Delta u=f(u)-f_{\Omega} f(u) d x, & x \in \Omega, t>0, \\ \frac{\partial u}{\partial n}=0, & x \in \partial \Omega, t>0, \\ u(x, 0)=u_{0}(x), & x \in \Omega .\end{cases}
$$

Here, $\Omega$ is a bounded smooth domain in $\mathbf{R}^{n}(n \geq 1)$ with $|\Omega|$ denoting its Lebesgue measure, $n$ is the outer normal vector of $\partial \Omega$, and the function $f(u)$ is usually taken to be a power of $u$. Problem (1.3) is also interesting in its own right, due to the fact that the comparison principle does not always hold for it. So it is often necessary to introduce some

(c) The Author(s) 2018. This article is distributed under the terms of the Creative Commons Attribution 4.0 International License (http://creativecommons.org/licenses/by/4.0/), which permits unrestricted use, distribution, and reproduction in any medium, provided you give appropriate credit to the original author(s) and the source, provide a link to the Creative Commons license, and indicate if changes were made. 
new techniques. The negative solutions of (1.3) with $f(u)=|u|^{p}$ are studied in [1]. When $f(u)=u|u|^{p}$ and $\int_{\Omega} u d x>0$ are studied in [2], the non-global existence result is discussed by using a convexity argument and under the energy condition

$$
E\left(u_{0}\right)=\int_{\Omega}\left[\frac{1}{2}\left|\nabla u_{0}\right|^{2}-\frac{1}{p+1}\left|u_{0}\right|^{p+1}\right] d x \leq-C .
$$

When $\int_{\Omega} u d x=0$, their conclusion can be found in [3, 4].

Further, the nonlocal $p$-Laplace equation

$$
\begin{cases}u_{t}-\operatorname{div}\left(|\nabla u|^{p-2} \nabla u\right)=u^{q}-f_{\Omega} u^{q} d x, & x \in \Omega, t>0, \\ \frac{\partial u}{\partial n}=0, & x \in \partial \Omega, t>0, \\ u(x, 0)=u_{0}(x), & x \in \Omega,\end{cases}
$$

is given in [5, 6], where a critical blow-up solution is determined by $q$ and the sign of the initial energy. In particular, the conditions under which the changing sign solutions vanish in finite time are obtained. At the same time, a thin-film has also received much attention, and it reads as follows:

$$
\begin{cases}u_{t}+u_{x x x x}=|u|^{p-1} u-f_{\Omega} u^{p-1} u d x, & x \in \Omega, t>0, \\ u_{x}=u_{x x x}=0, & x \in \partial \Omega, t>0, \\ u(x, 0)=u_{0}(x), & x \in \Omega .\end{cases}
$$

We can find it in $[7,8]$, where the threshold results of global existence and non-existence for the sign-changing weak solutions are given by the potential well method. Further, the conditions under which the global solutions become extinct in finite time are obtained and the asymptotic behavior of non-extinct solutions is studied.

To our knowledge, there have been few works concerned with the blow-up and extinction for the nonlocal parabolic equation with logarithmic nonlinearity. It is a remarkable fact that a nonlocal parabolic equation with logarithmic nonlinearity does not admit the usual maximum principle and the comparison principle. Because of this main difficulty, some most effective methods, such as the method of upper and lower solutions, are not valid here anymore. Inspired by the ideas in [9-18], the threshold results for the global existence and blow-up of the weak solutions are given by the potential well method, the classical Galerkin method, and the logarithmic Sobolev inequality. Further we discuss the non-extinction properties and the asymptotic behavior of the global solutions.

On the other hand, to handle logarithmic nonlinear term, we need the following logarithmic Sobolev inequality, which was introduced by $[10,19,20]$.

Proposition 1.1 $([10,19,20])$ Let $u$ be any function in $H^{1}\left(\mathbf{R}^{n}\right)$ and $a>0$ be any number. Then

$$
2 \int_{R^{n}}|u(x)|^{2} \log \left(\frac{|u(x)|}{\|u\|_{L^{2}\left(R^{n}\right)}}\right) d x+n(1+\log a)\|u\|_{L^{2}\left(R^{n}\right)}^{2} \leq \frac{a^{2}}{\pi} \int_{R^{n}}|\nabla u(x)|^{2} d x .
$$


For $u \in H_{0}^{1}(\Omega)$, we can define $u(x)=0$ for $x \in \mathbf{R}^{n} \backslash \Omega$. Then $u \in H^{1}\left(\mathbf{R}^{n}\right)$, that is to say, for a general domain $\Omega$, we have the following logarithmic Sobolev inequality:

$$
2 \int_{\Omega}|u(x)|^{2} \log \left(\frac{|u(x)|}{\|u\|_{L^{2}(\Omega)}}\right) d x+n(1+\log a)\|u\|_{L^{2}(\Omega)}^{2} \leq \frac{a^{2}}{\pi} \int_{\Omega}|\nabla u(x)|^{2} d x,
$$

where $u$ is any function in $H^{1}(\Omega), a>0$ is any number.

To state our main results, we need the following definitions.

Definition 1.1 (Weak solution) A function $u(x, t)$ is called a weak solution of (1.1), if $u \in$ $L^{\infty}\left(0, T ; H^{2}(\Omega)\right), u_{t} \in L^{2}\left(0, T ; L^{2}(\Omega)\right)$, and satisfies $(1.1)$ in the distribution sense, i.e.,

$$
\left(u_{t}, \varphi\right)_{2}+(\nabla u, \varphi)_{2}=(u \log |u|, \varphi)_{2}-\left(f_{\Omega} u \log |u|, \varphi\right)_{2}
$$

for any $\varphi \in H_{0}^{1}(\Omega), t \in(0, T)$, where $u(0, x)=u_{0}(x) \in H_{0}^{1}(\Omega)$, and $(\cdot, \cdot)_{2}$ means the inner product $(\cdot, \cdot)_{L^{2}(\Omega)}$.

Definition 1.2 (Finite time blow-up) Let $u(x, t)$ be a weak solution of (1.1). We call $u(x, t)$ blow-up in finite time if the maximal existence time $T$ is finite and

$$
\lim _{t \rightarrow T^{-}}\|u(\cdot, t)\|_{L^{2}(\Omega)}=+\infty .
$$

Definition 1.3 (Blow-up at $+\infty$ ) Let $u(x, t)$ be a weak solution of (1.1). We call $u(x, t)$ blow-up at $+\infty$ if the maximal existence time $T=+\infty$ and

$$
\lim _{t \rightarrow+\infty}\|u(\cdot, t)\|_{L^{2}(\Omega)}=+\infty
$$

For convenience, we introduce some notations here. Denote by $\|\cdot\|_{p}$ the $L^{p}(\Omega)$ norm for $1 \leq p \leq \infty$ and define

$$
\begin{aligned}
& J(u)=\frac{1}{2}\|\nabla u\|_{2}^{2}-\frac{1}{2} \int_{\Omega} u^{2} \log |u| d x+\frac{1}{4} \int_{\Omega} u^{2} d x, \\
& I(u)=\|\nabla u\|_{2}^{2}-\int_{\Omega} u^{2} \log |u| d x .
\end{aligned}
$$

Then it is obvious that

$$
\begin{aligned}
& J(u)=\frac{1}{2} I(u)+\frac{1}{4}\|u\|_{2}^{2}, \\
& \Phi=\left\{u \in H_{0}^{2}(\Omega) ; I(u)=0,\|u\|_{2}^{2} \neq 0\right\}, \\
& d=\inf _{u \in \Phi} J(u), \\
& M=\frac{1}{4}(2 \pi)^{\frac{n}{2}} e^{n} .
\end{aligned}
$$

From [10], we know $d \geq M$.

$$
W=\left\{u \in H_{0}^{1} ; I(u)>0, J(u)<d\right\} \cup\{0\}, \quad V=\left\{u \in H_{0}^{1} ; I(u)<0, J(u)<d\right\},
$$


and for $\delta>0$, we define

$$
\begin{aligned}
& I_{\delta}(u)=\delta\|\nabla u\|_{2}^{2}-\int_{\Omega} u^{2} \log |u| d x, \\
& N_{\delta}=\left\{u \in H_{0}^{2}(\Omega) ; I_{\delta}(u)=0,\|u\|_{2}^{2} \neq 0\right\}, \\
& d_{\delta}=\inf _{u \in N_{\delta}} J(u), \\
& W_{\delta}=\left\{u \in H_{0}^{1} ; I_{\delta}(u)>0, J(u)<d(\delta)\right\} \cup\{0\}, \\
& V_{\delta}=\left\{u \in H_{0}^{1} ; I_{\delta}(u)<0, J(u)<d(\delta)\right\} .
\end{aligned}
$$

The local existence of the weak solutions can be obtained via the standard parabolic theory. It is easy to obtain the following equality:

$$
\int_{0}^{t}\left\|u_{\tau}\right\|_{2}^{2} d \tau=J\left(u_{0}\right)-J(u), \quad 0<t<T .
$$

Theorem 1.1 If $u_{0} \in H_{0}^{1}, 0<J\left(u_{0}\right)<M$, and $I\left(u_{0}\right)>0$. Then problem (1.1) has a unique global weak solution, which does not blow up in finite time. Moreover,

$$
\|u\|_{2}^{2} \leq 2 e^{e^{t}} \log \frac{1}{2}\left\|u_{0}\right\|_{2}^{2}
$$

Theorem 1.2 Assume $J\left(u_{0}\right)<0$, then the solution $u(x, t)$ of problem (1.1) is blow-up at $+\infty$. Moreover, if $\left\|u_{0}\right\|_{2}^{2} \leq-2 J\left(u_{0}\right)$, the lower bound for blow-up rate can be estimated by

$$
\|u\|_{2}^{2} \geq 2\left\|u_{0}\right\|_{2}^{2}
$$

which is independent of $t$.

Theorem 1.3 Assume $0<J\left(u_{0}\right)<M$, then the solution $u(x, t)$ of problem (1.1) is non-extinct in finite time; moreover, $\|u\|_{2}^{2}$ can be estimated by

$$
\|u\|_{2}^{2} \geq\left\|u_{0}\right\|_{2}^{2} e^{-b t}, \quad b=n(1+\log \sqrt{2 \pi}) .
$$

$\|\cdot, \cdot\|_{2}$ means $\|\cdot, \cdot\|_{L^{2}(\Omega)}$.

Remark 1.1 For a nonlocal parabolic equation, $[5-8,21]$ get the results under appropriate conditions on blow-up in finite time. However, if $f(u)$ is the logarithmic nonlinear term $u \log |u|$ in (1.3), then Theorem 1.2 tells us that the weak solution is blow-up at $+\infty$.

Our paper is organized as follows. In Sect. 2, we establish new lemmas relative to the logarithmic nonlinear term. Next, in Sect. 3, we prove Theorem 1.1 (global existence and decay estimate). Finally, we give the proof of Theorems 1.2 (blow-up at $+\infty$ and the lower bound for blow-up rate) and 1.3 (non-extinction and estimate).

\section{Preliminaries}

In this section, we briefly collect some important properties of the functions and sets defined in the Introduction. 
Lemma 2.1 ([9]) Suppose that $\theta>0, \alpha>0, \beta>0$ and $h(t)$ is a nonnegative and absolutely continuous function satisfying $h^{\prime}(t)+\alpha h^{\theta}(t) \geq \beta$, then for $0<t<\infty$, it holds

$$
h(t) \geq \min \left\{h(0),\left(\frac{\beta}{\alpha}\right)^{\frac{1}{\theta}}\right\} .
$$

Lemma 2.2 If $0<J\left(u_{0}\right)<E_{1}=\frac{1}{4} e^{b}$, where $b=n(1+\log \sqrt{2 \pi})$, then there exists a positive constant $\alpha_{2}>\alpha_{1}$ such that

$$
\|u\|_{2} \geq \alpha_{2} .
$$

Proof Using the logarithmic Sobolev inequality in Proposition 1.1, for any $a>0$, we have

$$
\begin{aligned}
J(u) & =\frac{1}{2}\|\nabla u\|_{2}^{2}-\frac{1}{2} \int_{\Omega} u^{2}\left(\log \frac{|u|}{\|u\|_{2}}+\log \|u\|_{2}\right) d x+\frac{1}{4}\|u\|_{2}^{2}, \\
& \geq\left(\frac{1}{2}-\frac{a^{2}}{4 \pi}\right)\|\nabla u\|_{2}^{2}+\frac{1}{4} n(1+\log a)\|u\|_{2}^{2}-\frac{1}{2} \log \|u\|_{2}\|u\|_{2}^{2}+\frac{1}{4}\|u\|_{2}^{2} .
\end{aligned}
$$

Let $a=\sqrt{2 \pi}$ in (2.2), we have

$$
J(u) \geq\left(\frac{1}{4} n(1+\log a)-\frac{1}{2} \log \|u\|_{2}+\frac{1}{4}\right)\|u\|_{2}^{2} .
$$

Denote $\alpha=\|u\|_{2}, b=n(1+\log \sqrt{2 \pi})$, we have

$$
h(\alpha)=\left(\frac{b}{4}-\frac{\log \alpha}{2}+\frac{1}{4}\right) \alpha^{2} .
$$

Let

$$
h^{\prime}\left(\alpha_{1}\right)=0, \quad E_{1}=h\left(\alpha_{1}\right) .
$$

Furthermore, we get $h(\alpha)$ is increasing in $\left(0, \alpha_{1}\right)$ and decreasing in $\left(\alpha_{1}, \infty\right)$. Since $J\left(u_{0}\right)<E_{1}$, there exists a positive constant $\alpha_{2}>\alpha_{1}$ such that $J\left(u_{0}\right)=h\left(\alpha_{2}\right)$. Let $\alpha_{0}=\left\|u_{0}\right\|_{2}$, from (2.3) and (2.4), we have

$$
h\left(\alpha_{0}\right) \leq J\left(u_{0}\right) .
$$

Since $\alpha_{0}, \alpha_{2} \geq \alpha_{1}$, we get $\alpha_{0} \geq \alpha_{2}$, so (2.1) holds for $t=0$.

To prove (2.1) for $t>0$, we assume the contrary that $\|u(\cdot, t)\|_{2}<\alpha_{2}$ for some $t_{0}>0$. By the continuity of $\|u(\cdot, t)\|_{2}$ and $\alpha_{1}<\alpha_{2}$, we may choose $t_{0}$ such that $\left\|u\left(\cdot, t_{0}\right)\right\|_{2}>\alpha_{1}$, then it follows from (2.3)

$$
J\left(u_{0}\right)=h\left(\alpha_{2}\right)<h\left(\left\|u\left(\cdot, t_{0}\right)\right\|_{2}\right) \leq J(u)\left(t_{0}\right),
$$

which contradicts the fact that $J(u)$ is nonincreasing in $t$ by (1.12), so (2.1) is true. 
Lemma 2.3 Let $H(u)=E_{1}-J(u), J\left(u_{0}\right)<E_{1}$, then $H(u)$ satisfies the following estimates:

$$
0<H\left(u_{0}\right) \leq H(u) .
$$

Proof It is obvious that $H(u)$ is nondecreasing in $t$, by (1.12), then it follows from $J\left(u_{0}\right)<E_{1}$ that

$$
H(u) \geq H\left(u_{0}\right)=E_{1}-J\left(u_{0}\right)>0 .
$$

Remark 2.1 From Lemma 2.4 in [10], $M$ can be obtained by the definition of $d$, but in this paper, $E_{1}=h\left(\alpha_{1}\right), \alpha_{1}=e^{\frac{b}{2}}$, so we can get from (2.4)

$$
E_{1}=\frac{1}{4} e^{b}=M
$$

\section{Proofs of Theorem 1.1}

Proof of Theorem 1.1 We choose $w_{j}(x)$ to be the orthogonal basis of $H_{0}^{1}$ and construct the following approximate solution $u_{m}(x, t)$ of problem (1.1):

$$
u_{m}(x, t)=\sum_{j=1}^{m} g_{i}(t) w_{j}(x), \quad \text { for } m=1,2, \ldots
$$

which satisfies

$$
\begin{aligned}
& \left(u_{m t}, w_{s}\right)_{2}+\left(\nabla u_{m}, \nabla w_{s}\right)_{2}=\left(u_{m} \log \left|u_{m}\right|, w_{s}\right)_{2}-\left(f_{\Omega} u_{m} \log \left|u_{m}\right|, w_{s}\right)_{2} \\
& u_{m}(x, 0)=\sum_{j=1}^{m} a_{j} w_{j}(x) \rightarrow u_{0}
\end{aligned}
$$

and $(\cdot, \cdot)_{2}$ means the inner product $(\cdot, \cdot)_{L^{2}(\Omega)}$.

Next we need some a priori estimates on the approximate solutions $u_{m}$, multiplying by $g_{s}(t)$ and summing for $s$, for sufficiently large $m$. From (1.11), we obtain

$$
\int_{0}^{t}\left\|u_{m \tau}\right\|_{2}^{2} d \tau+J\left(u_{m}(t)\right)=J\left(u_{m}(0)\right)<M \leq d, \quad 0 \leq t<\infty .
$$

From the proof of Theorem 1.1 in [10], we have

$$
\begin{aligned}
& \left\|u_{m}\right\|_{2}^{2}<4 M, \\
& \left\|\nabla u_{m}\right\|_{2}^{2}<C_{M}, \\
& \int_{\Omega}\left(u_{m} \log \left|u_{m}\right|\right)^{2} d x \leq e^{-2}|\Omega|+\left(\frac{n-2}{2}\right)^{2} S^{2 *}\left\|\nabla u_{m}\right\|_{2}^{2 *} \leq C^{\prime} M,
\end{aligned}
$$

where $S$ is the best constant of the Sobolev embedding $H_{0}^{1}(\Omega) \hookrightarrow L^{\frac{2 n}{n-2}}(\Omega)$. 
Denote $\rightarrow\left(w^{*}\right)$ as the weakly star convergence. Therefore, we obtain a subsequence of $u_{m}$, which is still denoted by itself, our sending $m \rightarrow \infty$,

$$
\begin{aligned}
& u_{m} \rightarrow u\left(w^{*}\right) \quad \text { in } L^{\infty}\left(0, \infty ; H_{0}^{1}(\Omega)\right) \text { in } \Omega \times[0,+\infty), \\
& u_{m t} \rightarrow u_{t}\left(w^{*}\right) \quad \text { in } L^{2}\left(0, \infty ; L^{2}(\Omega)\right), \\
& u_{m} \log \left|u_{m}\right| \rightarrow u \log |u|\left(w^{*}\right) \quad \text { in } L^{\infty}\left(0, \infty ; L^{2}(\Omega)\right), \\
& f_{\Omega} u_{m} \log \left|u_{m}\right| \rightarrow f_{\Omega} u \log |u|\left(w^{*}\right) \quad \text { in } L^{\infty}\left(0, \infty ; L^{2}(\Omega)\right) .
\end{aligned}
$$

Hence, we have

$$
\left(u_{t}, w_{s}\right)_{2}+\left(\nabla u, \nabla w_{s}\right)_{2}=\left(u \log |u|, w_{s}\right)_{2}-\left(f_{\Omega} u \log |u|, w_{s}\right)_{2}
$$

Next, we prove the uniqueness of the weak solution. Suppose that problem (1.1) admits two weak solutions $u_{1}$ and $u_{2}$, set $w=u_{1}-u_{2}$, and $w$ solves

$$
\left\{\begin{array}{l}
w_{t}-\Delta w=f\left(u_{1}\right)-f\left(u_{2}\right), \quad(x, t) \in \Omega \times(0 . T) \\
\frac{\partial w}{\partial n}=0, \quad(x, t) \in \Omega \times(0 . T), \\
w(x, 0)=0, \quad x \in \Omega
\end{array}\right.
$$

where $f(u)=u \log |u|-f_{\Omega} u \log |u|$. Multiplying both sides of the first equation of (3.5) by $w$ and integrating the result over $\Omega \times(0, t)$, we have

$$
\begin{aligned}
\int_{0}^{t} & \int_{\Omega}\left(w_{t} w+|\nabla w|^{2}\right) d x d s \\
= & \int_{0}^{t} \int_{\Omega}\left(u_{1} \log \left|u_{1}\right|-u_{2} \log \left|u_{2}\right|\right) w d x d s \\
& -\int_{0}^{t} \int_{\Omega}\left(f_{\Omega} u_{1} \log \left|u_{1}\right|-u_{2} \log \left|u_{2}\right|\right) w d x d s \\
= & \int_{0}^{t} \int_{\Omega}\left(u_{1} \log \left|u_{1}\right|-u_{2} \log \left|u_{2}\right|\right) w d x d s \\
\leq & C \int_{0}^{t} \int_{\Omega} w^{2} d x d s,
\end{aligned}
$$

where $C$ is a positive constant.

Noticing that $w(x, 0)=0$, we have

$$
\int_{\Omega} w^{2}(x, t) d x \leq C \int_{0}^{t} \int_{\Omega} w^{2}(x, t) d x d t
$$

Using Gronwall's inequality, we get that

$$
\int_{\Omega} w^{2}(x, t) d x=0
$$

Thus $w=0$ a.e. in $\Omega \times(0, T)$. 
We define $M(t)=\frac{1}{2}\|u\|_{2}^{2}$, then

$$
M^{\prime}(t)=\left(u_{t}, u\right)_{2}=\left((u \log |u|, u)_{2}-\left(f_{\Omega} u \log |u| d x, u\right)_{2}+\|\nabla u\|_{2}^{2}\right)=-I(u) .
$$

By using (1.9) and Proposition 1.1, we know

$$
I(u) \geq\left(\frac{n(2+\log (2 \pi)}{4}-\log \|u\|_{2}^{2}\right)\|u\|_{2}^{2},
$$

where $a=\sqrt{2 \pi}$.

From (3.6) and (3.7)

$$
M(t) \log M(t)-M^{\prime}(t) \geq\left(\frac{n(2+\log (2 \pi)}{4}-\log \|u\|_{2}^{2}\right)\|u\|_{2}^{2} \geq 0
$$

we have

$$
(\log M(t))^{\prime} \leq \log M(t)
$$

This means

$$
M(t) \leq e^{e^{t}} \log \frac{1}{2}\left\|u_{0}\right\|_{2}^{2}
$$

then

$$
\|u\|_{2}^{2} \leq 2 e^{e^{t}} \log \frac{1}{2}\left\|u_{0}\right\|_{2}^{2} \quad \text { for all } t \geq 0
$$

which implies that $u(x, t)$ does not blow up in finite time.

\section{Proofs of Theorems 1.2 and 1.3}

Proof of Theorem 1.2 By the definition of $J(u), J\left(u_{0}\right)<0$ and (1.12), $M(t)$ satisfies

$$
\begin{aligned}
M^{\prime}(t) & =\int_{\Omega} u \cdot u_{t} d x \\
& =\int_{\Omega} u\left(\Delta u+u \log |u|-f_{\Omega} u \log |u|\right) d x \\
& =\int_{\Omega}\left(-|\nabla u|^{2}+u^{2} \log |u|\right) d x \\
& \geq-2 J(u) \\
& \geq 2 \int_{0}^{t}\left\|u_{\tau}\right\|_{2}^{2} d \tau .
\end{aligned}
$$

And by the definition of weak solution, we know that $u \in L^{\infty}\left(0, T ; H^{2}(\Omega)\right)$. For any $t_{0}>0$, we claim that

$$
\int_{0}^{t_{0}}\left\|u_{\tau}\right\|_{2}^{2} d \tau>0
$$


Otherwise, there exists $t_{0}>0$ such that $\int_{0}^{t_{0}}\left\|u_{\tau}\right\|_{2}^{2} d \tau=0$, and hence $u_{t}=0$ for a.e. $(x, t) \in$ $\Omega \times\left(0, t_{0}\right)$. Then it follows from (4.1) that

$$
-|\nabla u|^{2}+u^{2} \log |u|=0
$$

for a.e. $t \in\left(0, t_{0}\right)$, and then we get from (1.9)

$$
J(u)=\int_{\Omega} \frac{1}{4} u^{2} d x .
$$

Combining it with $J(u) \leq J\left(u_{0}\right) \leq 0$, we obtain $\|u\|_{2}=0$ for all $t \in\left[0, t_{0}\right]$, which contradicts the definition of $u$. Then (4.2) follows.

Fix $t_{0}>0$ and let $\delta=\int_{0}^{t}\left\|u_{\tau}\right\|_{2}^{2} d \tau$, then we know that $\delta$ is a positive constant. Integrating (4.1) over $\left(t_{0}, t\right)$, we obtain

$$
M(t) \geq M\left(t_{0}\right)+\int_{t_{0}}^{t} \int_{0}^{s}\left\|u_{\tau}\right\|_{2}^{2} d \tau d s \geq \delta\left(t-t_{0}\right) .
$$

Hence,

$$
\lim _{t \rightarrow \infty} H^{\prime}(t)=\lim _{t \rightarrow \infty} M(t)=\infty .
$$

And from (4.1), we know

$$
\begin{aligned}
M^{\prime}(t) & =\int_{\Omega} u \cdot u_{t} d x \\
& =\int_{\Omega} u\left(\Delta u+u \log |u|-f_{\Omega} u \log |u|\right) d x \\
& =\int_{\Omega}\left(-|\nabla u|^{2}+u^{2} \log |u|\right) d x \\
& =-2 J(u)+\frac{1}{2}\|u\|_{2}^{2} \\
& \geq-\frac{1}{2}\|u\|_{2}^{2}-2 J\left(u_{0}\right) .
\end{aligned}
$$

By using Lemma $2.1, J\left(u_{0}\right)<0$, and $\left\|u_{0}\right\|_{2}^{2} \leq-2 J\left(u_{0}\right)$, we have

$$
\begin{aligned}
M(t) & \geq \min \left\{\left\|u_{0}\right\|_{2}^{2},-2 J\left(u_{0}\right)\right\} \\
& \geq\left\|u_{0}\right\|_{2}^{2},
\end{aligned}
$$

which means (1.13) is true.

Proof of Theorem 1.3 We define $\beta=\frac{\alpha_{2}}{\alpha_{1}}>1, M(t)=\frac{1}{2}\|u\|_{2}^{2}$. Then, by the definitions of $J(u)$, $M(t)$, and Lemma 2.3, we have

$$
\begin{aligned}
M^{\prime}(t) & =\int_{\Omega} u \cdot u_{t} d x \\
& =\int_{\Omega} u\left(\Delta u+u \log |u|-f_{\Omega} u \log |u|\right) d x
\end{aligned}
$$




$$
\begin{aligned}
& =\int_{\Omega}\left(-|\nabla u|^{2}+u^{2} \log |u|\right) d x \\
& =-2 J(u)+\frac{1}{2} \int_{\Omega} u^{2} d x \\
& =-2 E_{1}+H(u)+\frac{1}{2} \int_{\Omega} u^{2} d x .
\end{aligned}
$$

By using (2.1) and (2.2) in Lemma 2.2, we get

$$
\begin{aligned}
E_{1} & =\left(\frac{1}{4} b-\frac{1}{2} \log \frac{\alpha_{2}}{\beta}+\frac{1}{4}\right) \frac{\alpha_{2}^{2}}{\beta^{2}} \\
& \leq\left(\frac{1}{4} b+\frac{1}{4}\right) \frac{\alpha_{2}^{2}}{\beta^{2}} \\
& \leq\left(\frac{1}{4} b+\frac{1}{4}\right)\|u\|_{2}^{2} .
\end{aligned}
$$

Then it follows from (4.6), (4.7), and Lemma 2.3 that

$$
\begin{aligned}
M^{\prime}(t) & \geq-2 E_{1}+\frac{1}{2}\|u\|_{2}^{2} \\
& \geq-\frac{1}{2} b\|u\|_{2}^{2} \\
& \geq-b M(t),
\end{aligned}
$$

which means (1.14) is true.

\section{Acknowledgements}

We would like to thank the referees for their valuable comments and suggestions to improve our paper.

\section{Funding}

Project supported by the National Natural Science Foundation of China (Grant No. 11571093 and No. 11471164).

\section{Availability of data and materials}

Data sharing not applicable to this article as no data sets were generated or analyzed during the current study.

\section{Competing interests}

The authors declare that they have no competing interests.

\section{Authors' contributions}

This work was carried out in collaboration between both authors. ZY designed the study and guided the research. LY performed the analysis and wrote the first draft of the manuscript. ZY and LY managed the analyses of the study. Both authors read and approved the final manuscript.

\section{Author details}

${ }^{1}$ Institute of Mathematics, School of Mathematics Science, Nanjing Normal University, Nanjing, China. ${ }^{2}$ School of Teacher Education, Nanjing Normal University, Nanjing, China.

\section{Publisher's Note}

Springer Nature remains neutral with regard to jurisdictional claims in published maps and institutional affiliations.

Received: 2 March 2018 Accepted: 26 July 2018 Published online: 02 August 2018

\section{References}

1. Wang, M., Wang, Y.: Properties of positive solutions for non-local reaction-diffusion problems. Math. Methods Appl. Sci. 19(14), 1141-1156 (1996)

2. Hu, B., Yin, H.M.: Semilinear parabolic equations with prescribed energy. Rend. Circ. Mat. Palermo 44(3), 479-505 (1995) 
3. El Soufi, A., Jazar, M., Monneau, R.: A Gamma-convergence argument for the blow-up of a non-local semilinear parabolic equation with Neumann boundary conditions. Ann. Inst. Henri Poincaré, Anal. Non Linéaire 24(1), 17-39 (2007)

4. Budd, C.J., Dold, J.W., Galaktionov, V.A.: Global blow-up for a semilinear heat equation on a subspace. Proc. R. Soc. Edinb., Sect. A, Math. 145(5), 893-923 (2015)

5. Qu, C., Bai, X., Zheng, S.: Blow-up versus extinction in a nonlocal $p$-Laplace equation with Neumann boundary conditions. J. Math. Anal. Appl. 412(1), 326-333 (2014)

6. Qu, C., Liang, B.: Blow-up in a slow diffusive-Laplace equation with the Neumann boundary conditions. Abstr. Appl. Anal. 2013, Article ID 643819 (2013)

7. Cao, Y., Liu, C.: Global existence and non-extinction of solutions to a fourth-order parabolic equation. Appl. Math. Lett. $61,20-25(2016)$

8. Qu, C., Zhou, W.: Blow-up and extinction for a thin-film equation with initial-boundary value conditions. J. Math. Anal. Appl. 436(2), 796-809 (2016)

9. Guo, B., Gao, W.J.: Non-extinction of solution for a fast diffusive $p$-Laplace equation with Neumann boundary conditions. J. Math. Anal. Appl. 422, 1527-1531 (2015)

10. Chen, H., Luo, P., Liu, G.: Global solution and blow-up of a semilinear heat equation with logarithmic nonlinearity. J. Math. Anal. Appl. 422(1), 84-98 (2015)

11. Yacheng, L., Junsheng, Z.: On potential wells and applications to semilinear hyperbolic equations and parabolic equations. Nonlinear Anal., Theory Methods Appl. 64(12), 2665-2687 (2006)

12. Castillo, R., Loayza, M.: A semilinear parabolic problem with variable reaction on a general domain. Comput. Math Appl. 74(3), 351-359 (2017)

13. Levine, H.A.: The role of critical exponents in blowup theorems. SIAM Rev. 32(2), 262-288 (1990)

14. Polacik, P., Quittner, P., Souplet, P.: Singularity and decay estimates in superlinear problems via Liouville-type theorems, I: elliptic equations and systems. Duke Math. J. 139(3), 555-579 (2007)

15. Polacik, P., Quittner, P., Souplet, P.: Singularity and decay estimates in superlinear problems via Liouville-type theorems. Part II: parabolic equations. Indiana Univ. Math. J. 56(2), 879-908 (2007)

16. Quittner, P.: Threshold and strong threshold solutions of a semilinear parabolic equation. Adv. Differ. Equt. 22(7/8), 433-456 (2017)

17. Shahmurov, R.: Solution of Dirichlet and Neumann problems for a modified Helmholtz equation in Besov spaces on an annulus. J. Differ. Equ. 249(3), 526-550 (2010)

18. Shahmurov, R.: On strong solutions of a Robin problem modelling heat conduction in materials with corroded boundary. Nonlinear Anal., Real World Appl. 13(1), 441-451 (2012)

19. Lieb, E.H., Loss, M.: Analysis, Graduate Studies in Mathematics, vol. 14. Am. Math. Soc., Providence (2001)

20. Gross, L.: Logarithmic Sobolev inequalities. Am. J. Math. 97, 1061-1083 (1976)

21. Deng, K., Levine, H.A.: The role of critical exponents in blow-up theorems: the sequel. J. Math. Anal. Appl. 243(1), $85-126(2000)$

\section{Submit your manuscript to a SpringerOpen ${ }^{\circ}$ journal and benefit from:}

- Convenient online submission

- Rigorous peer review

- Open access: articles freely available online

- High visibility within the field

- Retaining the copyright to your article

Submit your next manuscript at $\gg$ springeropen.com 